\title{
Serratus Plane Block. Above or Beneath the Serratus muscle? Initial evaluation of efficacy and safety.
}

\author{
Kaushal Kumar, Sanjit Naskar, Debesh Bhoi \\ All India Institute of Medical Sciences, Dept of Anaesthesiology, New Delhi, India
}

\section{Background}

$\checkmark$ Chronic postsurgical pain is very common after Breast surgery and known as Post mastectomy pain syndrome (PMPS).

$\checkmark$ Incidence of PMPS is more in case of inadequate postoperative pain management.

$\checkmark$ Moreover, there is more recurrence of PMPS with use of higher dose of opioids.

- Hence, An ideal postoperative pain strategy in case of Breast surgery would be an Opioid sparing multimodal pain program.

- Blanco et al in 2013 described a novel regional approach to block anterior and lateral cutaneous branches of the intercostal nerves in the mid-axillary line both superficial and underneath the Serratus muscle in 4 volunteers.

$\checkmark$ Diéguez and colleagues confirmed the efficacy of the deeper block between the serratus and external intercostal muscle for postoperative pain management after Breast surgery in a sample of 30 patients.

\section{Objective}

- Initial evaluation of efficacy and safety of the serratus anterior plane (SAP) block, both superficial and deep to the serratus muscle in patients undergoing breast surgery.

\section{Sample}

- Medical records and anaesthesia charts of 20 patients who underwent Modified Radical mastectomy with axillary clearance and received SAP block between September to November 2015 were reviewed.

- All patients had received SAP block with $30 \mathrm{ml}$ of $0.375 \%$ Ropivacaine under ultrasound guidance in a plane above or beneath the serratus muscle as described by Blanco.

$\checkmark$ Clinical efficacy and safety of both the techniques was assessed by the use of intraoperative opioids and complication if any, in anaesthesia chart and by assessing the numerical rating pain scores and the need for rescue analgesia in postoperative notes.

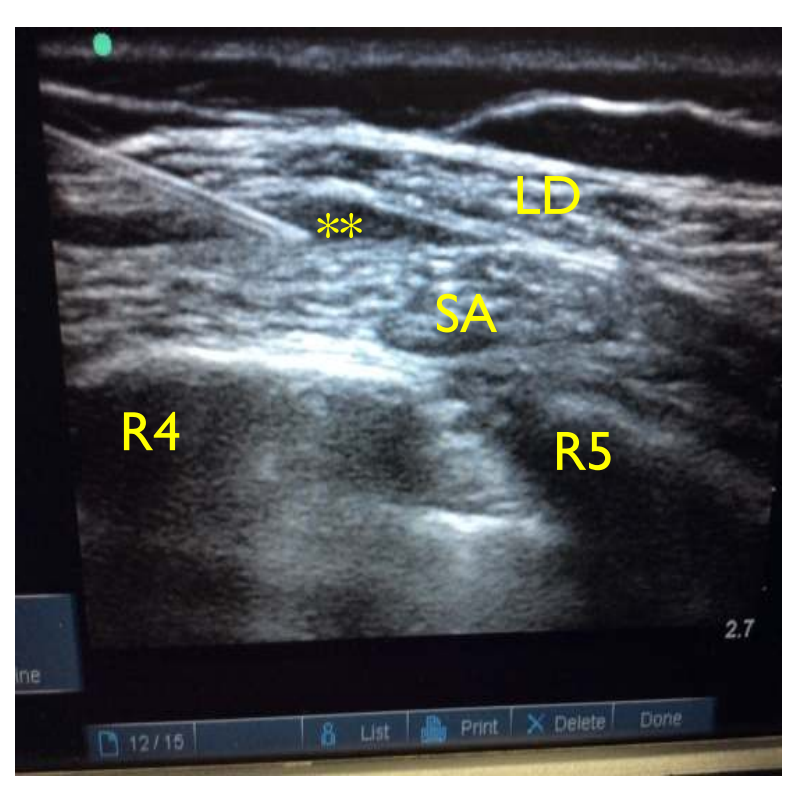

Superficial SAP Block

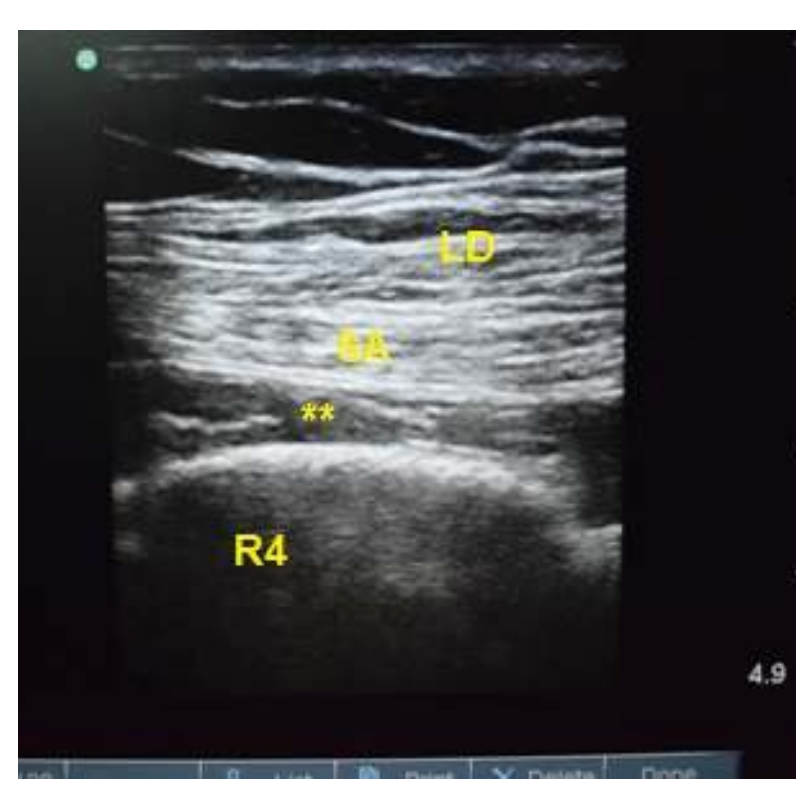

Deep SAP Block

\section{References}

Blanco R, Parras T, McDonnell JG, Prats-Galino, A. (2013), Serratus plane block: a novel ultrasound-guided thoracic wall nerve block. Anaesthesia.2013,68:1107-13.

$\checkmark$ Diéguez P, Fajardo M, López S, Alfaro P, Pensado A. Ultrasoundassisted approach to blocking the intercostal nerves in the midaxillary line for non- reconstructive breast and axilla surgery. Revista Espanola de Anestesiologia Reanimacion 2013.04.002.

Results
Total no. of cases : 20
Superficial SAP Block : 7
Deep SAP Block : 13

Intraoperative Opioid Requirement

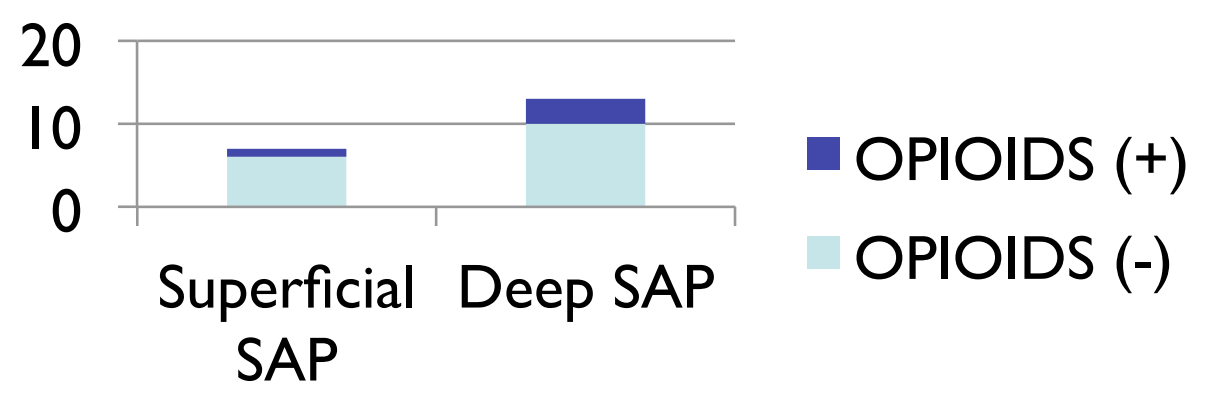

Postoperative pain (NRS) scores (Mean)

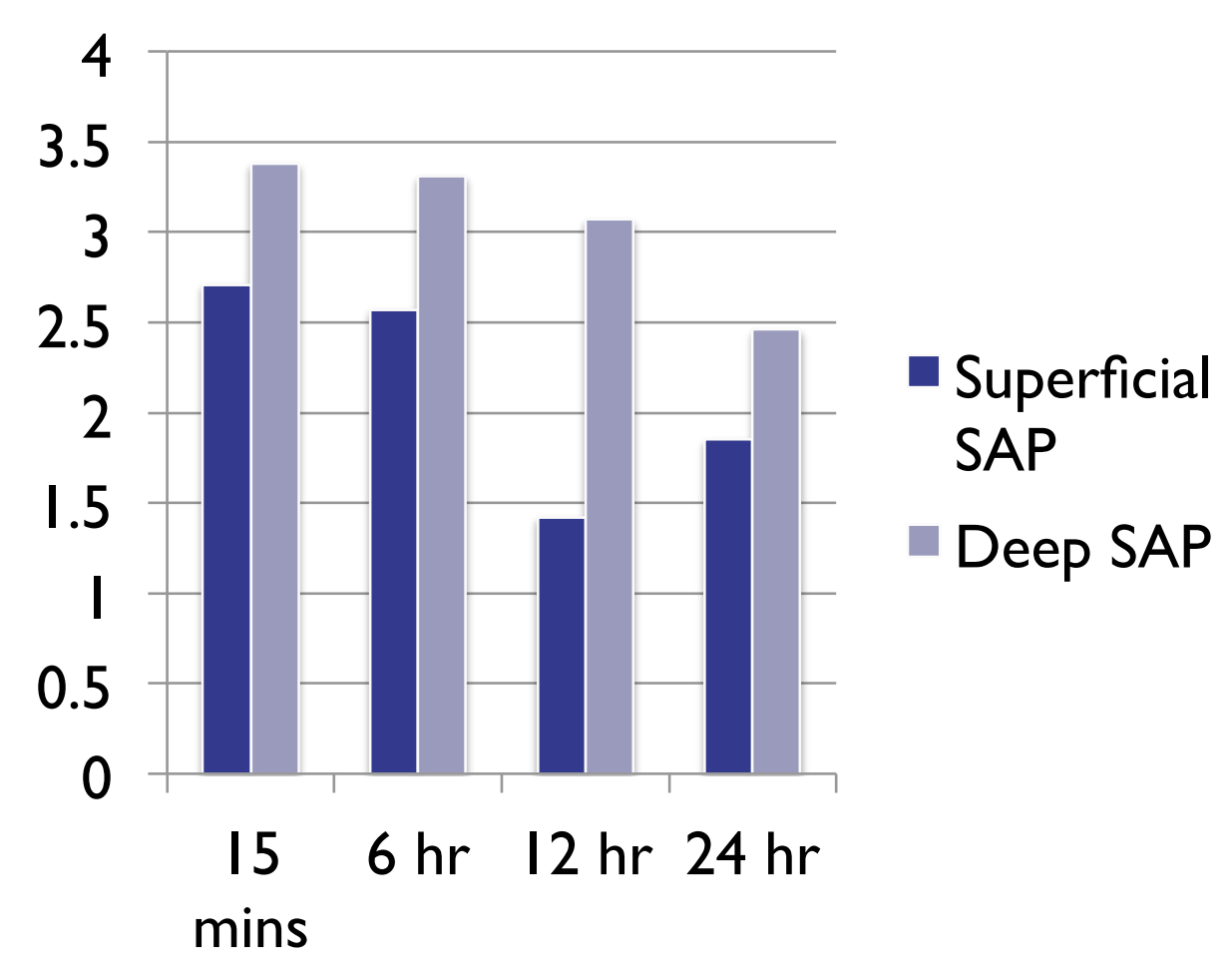

$\checkmark$ Post operative pain scores (NRS) were below 4/10 in both the blocks and was well managed with intravenous Paracetamol and Diclofenac.

$\checkmark 1$ patient who received a deep SAP block had a pain score of 5 or more in a follow-up period of 24 hours and received intravenous tramadol.

$\checkmark$ None of the patients had any complications, but we had technical difficulty in deep block due to excessive axillary fat in 1 patient.

\section{Discussion}

SAP block is a simple regional technique, which provides good opioid sparing analgesia in a multimodal pain management program in Modified Radical mastectomy with axillary node clearance.

- Post operative pain scores were more or less similar in both blocks, however, the superficial block has slight comparative advantage over the deep block especially in the late postoperative period after 6 hours. This corroborates with the Blanco's study in which the superficial SAP block had more dermatomal distribution of sensory loss (T2-T9) as compared to the deep block(T2-T6,7) and longer duration of the superficial block.

$\checkmark$ Regarding the preference over the plane of the block either above or beneath the serratus muscle, It seems the superficial plane has slight advantage over the deep plane in terms of efficacy, ease of administration and safety of the block. Though, larger RCT are required for the confirmation of the same.

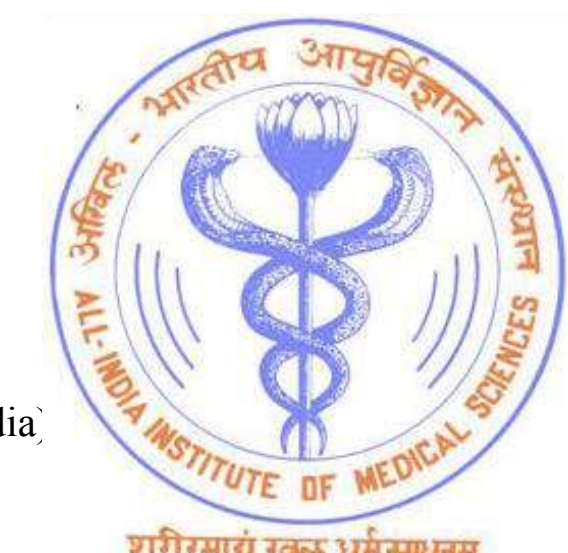

